Check for updates

Cite this: RSC Adv., 2017, 7, 49542

Received 6th September 2017 Accepted 12th October 2017

DOI: $10.1039 / c 7 r a 09924 j$

rsc.li/rsc-advances

\section{Efficient and stable white fluorescent carbon dots and CD-based glass thin-films via screen-printing technology for use in W-LEDs $\uparrow$}

\author{
Yijun Zhang, Jiali Feng, Meiling He, Jutao Jiang, Tao Xu, Lei Zhou, Wei Chen, \\ Weidong Xiang (iD * and Xiaojuan Liang (D) *
}

White fluorescent carbon dots (WCDs) were synthesized by combining red carbon dots (RCDs) with ethanol or $N$-( $\beta$-aminoethyl)- $\gamma$-aminopropyl methldimethoxy silane (AEAPMS) for the first time. Interestingly, the RCDs have excitation-independent behavior while the WCDs exhibit excitation-dependent behavior. These WCDs emit bright white light with quantum yields (QYs) of $50.8 \%$ and $57.1 \%$. Solvatochromism effects of the CDs were ascribed to the intermolecular charge transfer between the CDs and solvents. The solvents further increase the charge carrier density and induce the charge transfer. Investigation of the white fluorescence mechanism of the CDs revealed a multilevel emissive recombination channel, which might be from the distribution of diverse surface-state PL centers. Additionally, because the latter WCDs exhibit excellent optical properties and a great film-forming ability, workable white CD-based glass thin-films were constructed using facile screen-printing technology. Finally, the as-fabricated white light-emitting diodes (W-LEDs) exhibited an optimized color coordinate (CIE) of $(0.33,0.34)$, color rendering index (CRI) of 51 and correlated color temperature (CCT) of $6735 \mathrm{~K}$. Therefore, the WCDs reveal the strong feasibility of being used for lighting and displays.

\section{Introduction}

Carbon dots (CDs) have drawn attention due to their unique properties in various applications, for example, bioimaging, optoelectronic devices, photocatalysis and sensors..$^{1-5}$ Particularly, considerable efforts have been devoted to $\mathrm{CD}$-based materials in W-LEDs. ${ }^{4-9}$ It is interesting that CDs with the solvatochromism phenomenon of changing from blue to red in different solvents have been observed. Lin et al. reported that CDs prepared from $m$-phenylenediamine exhibit emission at 400-500 $\mathrm{nm}$ in aqueous solution and PVA. ${ }^{10}$ Wang and coworkers claimed that CDs synthesized from $p$-phenylenediamine emit various lights (green to red, from 511 to $615 \mathrm{~nm}$ ) in different solvents. ${ }^{11}$ Graphene quantum dots were also described to have different emissions from 475 to $515 \mathrm{~nm}$ in a few solvents. ${ }^{12}$ Yet, few literature studies have reported white light CDs in solvents. Besides, there are only several studies on CDs emitting white photoluminescence, which is expected for commercial applications of W-LEDs. ${ }^{7-9}$ Wang and coworkers developed a white-light CD solution and film illuminated with $407 \mathrm{~nm}$ light, and the highest QY of the CDs was 6\%.7 Chen's

College of Chemistry and Materials Engineering, Wenzhou University, Wenzhou 325035, China. E-mail: xiangweidong001@126.com; lxj6126@126.com; Fax: +86577-86689644; Tel: +86-577-86596013

† Electronic supplementary information (ESI) available. See DOI: $10.1039 / \mathrm{c} 7 \mathrm{ra09924j}$ group produced blue, orange and white emitting CDs (QY = $47 \%$ ) using a single-step pyrolysis route in $2012 .{ }^{8}$ Subsequently, Dang reported white emission CDs with a QY of $28.3 \%$ after treatment with KH570 using a one-step ultrasonic method. ${ }^{9}$ Furthermore, few studies have mentioned solid-state fluorescence of white CDs, such as white CD-based phosphors, ${ }^{13-16}$ and all of these CDs did not present pure white emission under an ultraviolet lamp. Even so, CDs suffer from a low QY and complicated preparation procedures. It is well-known that aggregation-induced luminescence quenching of CDs is the main obstacle for their application in the solid state. In other cases, white-light-emitting CDs are directly utilized with blue chips. ${ }^{17}$ Hence, effective methods should be proposed to preserve the photoluminescence of CDs in the solid state. Organosilane (AEAPMS) is a great passivator to avoid solid quenching of CDs because of the chain network structure. CDs can homogeneously disperse in AEAPMS to avoid aggregation. Meanwhile, silane-amine based materials have a good filmforming ability ${ }^{6,18}$ due to the large surface area and surface functional groups. Therefore, the obtained CDs dispersed in AEAPMS have favorable photostability. It is desirable for nitrogen-doping agents to endow nitrogen-doped CDs with a promising QY and photostability.

Herein, we report a facile and economic solvothermal route towards preparing red fluorescent carbon dots. Impressively, WCDs with high performances were achieved through passivation by ethyl alcohol and AEAPMS. AEAPMS was chosen as the 
solid state medium to avoid fluorescence quenching of the CDs. Moreover, direct white emission CD-based glass thin-films can be applied as an inexpensive color-changing layer in W-LEDs. These results demonstrate the promising application of WCDs in LEDs.

\section{Experimental}

RCDs were synthesized by a facile solvothermal method. First, ammonium citrate $(1 \mathrm{~g}, \mathrm{AC})$ was dispersed in $10 \mathrm{ml}$ of DMF and then transferred to a $20 \mathrm{ml}$ Teflon lined stainless-steel autoclave. The reactor was heated at $180^{\circ} \mathrm{C}$ for $3 \mathrm{~h}$, resulting in the RCDs. The products were purified with petroleum ether three times. For the WCDs, combining the RCDs $(0.8 \mathrm{ml})$ with ethyl alcohol $(10 \mathrm{ml})$ or $N$-( $\beta$-aminoethyl)- $\gamma$-aminopropyl methldimethoxy silane (AEAPMS) $(10 \mathrm{~mL})$ produced the white luminescent CDs, which are denoted as WCDs-1 and WCDs-2.

The WCDs-2 have a great film-forming ability. To investigate the application of WCDs-2 as phosphors in LEDs, a CD-based glass thin-film was fabricated. Firstly, the glass thin-film was prepared by screen-printing the WCDs- 2 solution on a glass plate (commercial glass) with a diameter of $50 \mathrm{~mm}$ and thickness of $0.2 \mathrm{~mm}$. The acquired hybrids were subsequently dried in air at $120^{\circ} \mathrm{C}$ to eliminate bubbles and solidify the film.

For the W-LEDs, the CD-based glass thin-film was cut into a $3.3 \times 2.8 \mathrm{~mm}$ cullet. Then, the WCD-based LEDs were constructed by integrating the glass film with a UV LED chip (395 $\mathrm{nm})$.

\subsection{Characterization}

The absorption spectrum was collected using a UV-2450 spectrometer. To confirm the microstructure and particle distribution of the CDs, a FEI Tecnai F20 transmission electron microscope operating at $200 \mathrm{kV}$ was adopted to acquire the transmission electron microscopy (TEM) and high-resolution TEM (HRTEM) images. A Horiba Jobin Yvon Fluromax-4P spectrophotometer equipped with absolute QY measurement apparatus was used to obtain the fluorescence spectra and PL QY. The QY measurements of the samples were conducted using an integrating sphere. Two $10 \mathrm{~mm}$ quartz cuvettes were used to separately fill an equal volume of the CD solution and pure solvent (alcohol and AEAPMS) for contrast. Subsequently, the WCD solution was excited with monochromatic light at the optimal excitation wavelength $(360 \mathrm{~nm})$. The fluorescence spectra were recorded in the ranges of $350-370 \mathrm{~nm}$ and $370-$ $710 \mathrm{~nm}$. Moreover, the fluorescence spectra of pure solvent (alcohol and AEAPMS) were recorded under identical conditions. Finally, fluorescence software (HORIBA Jobin Yvon Quantum Yield and Color Calculator) was used to calculate the QY according to the spectra of the WCD sample and pure solvent. To confirm the surface state and functional chemical groups of the CDs, both X-ray photoelectron spectra (XPS) and Fourier transform infrared (FTIR) spectra were recorded using an Axi Ultra DLD spectrometer with monochromatic $\mathrm{Al} \mathrm{K} \alpha$ as the excitation source and a Bruke Equinox 55 FTIR spectrometer in the range of $600-4000 \mathrm{~cm}^{-1}$, respectively. The luminescence properties of the LED device were determined with an integrating sphere (PMS-50, Everfine, China) to gain the CIE, CRI and CCT under a forward current of $20 \mathrm{~mA}$.

\section{Results and discussion}

RCDs have been synthesized by a facile solvothermal method using AC (ammonium citrate) as the C (carbon) source. A bright red solution was observed under a UV lamp (365 nm, Fig. S1 (ESI $\dagger)$ ). The as-obtained RCDs (Fig. 1(a) and (b)) exhibit a welldispersed and relatively homogeneous spherical shape with sizes in the range of 3-8 $\mathrm{nm}$. Clearly, the HRTEM micrograph of individual RCDs illustrates that the RCDs exhibit lattice fringes with a $d$-spacing of $0.21 \mathrm{~nm}$, corresponding to the (100) plane lattice of graphite. ${ }^{4}$ Similar crystallinity with lattices of $0.21 \mathrm{~nm}$ was also found in WCDs-1 (Fig. S2(b) (ESI $\dagger$ )) and WCDs-2 (Fig. S2(e) (ESI†)). Fig. S2(a)† shows that the WCDs-1 are welldispersed and highly monodispersed, along with having particle sizes of 2-10 $\mathrm{nm}$. Compared with the WCDs-2, the asprepared WCDs-1 tended to aggregate into "clusters" due to the polarity of ethyl alcohol, as shown in Fig. S2(a). $\dagger$ Fig. 1(c) gives the outline of the UV absorption spectra. The characteristic absorption peaks located at 230 and $340 \mathrm{~nm}$ correspond to the $\pi-\pi^{*}$ transitions of the $\mathrm{C}=\mathrm{C}$ and $\mathrm{C}=\mathrm{N}$ bonds in the highenergy region (200-350 nm). ${ }^{4-6}$ Interestingly, as presented in Fig. 1(c), the RCDs exhibited excitation-independent behavior. Generally, the excitation-dependent behavior of CDs may be derived from particles of different sizes in the sample and the distribution of the different surface states. ${ }^{19}$ However, the TEM images indicate that the size distribution of the RCDs ranges from 4 to $8 \mathrm{~nm}$, which is not greatly uniform. Therefore, the excitation-independent behavior reveals that the PL properties of the RCDs rely on the surface states rather than the morphology. Hence, the surface states of the RCDs should be rather uniform. To confirm the surface functional groups of the RCDs, XPS and FTIR measurements were implemented (Fig. 1(d)-(f)). As can be seen, three typical peaks, C 1s (285 $\mathrm{eV}), \mathrm{N} 1 \mathrm{~s}(400 \mathrm{eV})$, and $\mathrm{O} 1 \mathrm{~s}(531 \mathrm{eV})$, were observed. The C 1s band can be resolved into three peaks including $\mathrm{sp}^{2}$ carbons $(\mathrm{C}=\mathrm{C} / \mathrm{C}-\mathrm{C}, 284.5 \mathrm{eV}), \mathrm{sp}^{3}$ carbons $(\mathrm{C}-\mathrm{N}, 285.7 \mathrm{eV}$ ), and carbonyl carbons $(\mathrm{C}=\mathrm{O}, 287.7 \mathrm{eV})$, which is consistent with a previous report. ${ }^{20}$ The $\mathrm{N} 1 \mathrm{~s}$ band at $399.6 \mathrm{eV}$ is assigned to amino $\mathrm{N}$. The content of the nitrogen, oxygen, and carbon is detailed in Table S1. $\dagger$ The results further confirm that the incorporation of $\mathrm{N}$ in the CDs is responsible for the red luminescence. ${ }^{6}$ Besides, we have characterized the WCDs- 1 and WCDs- 2 with an XPS test. Similarly, as shown in Fig. S4, $\uparrow$ the spectra of the WCDs-2 display C 1s, N 1s, O 1s and Si 2p peaks at 285.0, 400.0, 531.0, and $101.0 \mathrm{eV}$, respectively, which are similar to those of the WCDs-1, as shown in Fig. S3. $\dagger$ The N 1s (Fig. S3(d) †) band peaks at $399.6 \mathrm{eV}$ represent amino N. The location of the peak of Si 2p (Fig. S4(d) $\dagger$ ) indicates the groups of Si-O-Si. As characterized by FTIR, the RCDs possess abundant hydrophilic groups like $\mathrm{O}-\mathrm{H}$ $\left(3468 \mathrm{~cm}^{-1}\right), \mathrm{N}-\mathrm{H}\left(3383-3163 \mathrm{~cm}^{-1}\right), \mathrm{C}-\mathrm{O}\left(1258 \mathrm{~cm}^{-1}\right)$ and $\mathrm{C}=\mathrm{O}\left(1628 \mathrm{~cm}^{-1}\right)$ on their surface. Besides, stretching vibrations of $\mathrm{C}-\mathrm{H}\left(2943 \mathrm{~cm}^{-1}\right), \mathrm{C}=\mathrm{N}\left(1655 \mathrm{~cm}^{-1}\right), \mathrm{C}-\mathrm{C}\left(1524 \mathrm{~cm}^{-1}\right)$, and $\mathrm{C}-\mathrm{N}$ (1388 and $1496 \mathrm{~cm}^{-1}$ ) bonds were observed., ${ }^{4,6,21}$ This 

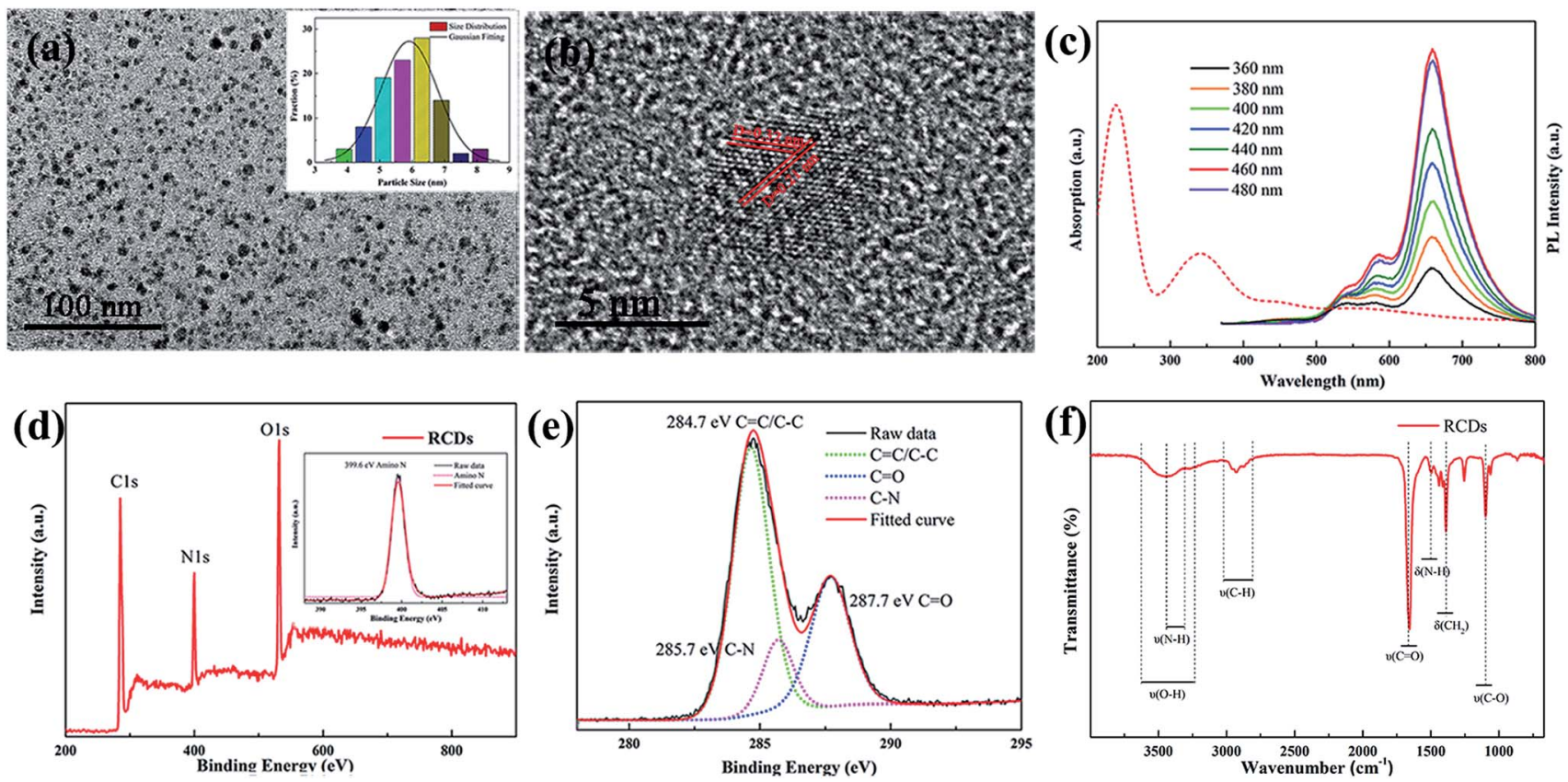

Fig. 1 TEM image (a), HRTEM image (b), and UV-vis absorption spectra and emission spectra (c) of the RCDs. The inset in (a) shows the particle size distribution histogram. The XPS full survey (d), a high-resolution XPS spectrum of $C$ 1s (e), and the FTIR spectrum of the RCDs (f). The inset in (d) shows a high-resolution XPS spectrum of $\mathrm{N}$ 1s of the RCDs.

suggests that the RCDs exhibit excellent water solubility so that the RCDs can be mutually solubilized with ethyl alcohol and AEAPMS. This is in good agreement with the result of the FTIR investigation of the WCDs-1 and WCDs-2, shown in Fig. S5. $\dagger$ Compared with the WCDs-1, we can observe strong peaks at about $1450,1070-1098$ and $790 \mathrm{~cm}^{-1}$ originating from the $-\mathrm{CH}_{2}$ bending, Si-O-Si stretching and $\mathrm{Si}-\mathrm{CH}_{2}$ stretching modes, respectively. The $\mathrm{Si}-\mathrm{O}-\mathrm{Si}$ structure is a great passivator to avoid quenching of the CDs because of the chain network structure, which guarantees the stability of the WCDs-2.

Furthermore, the PL emission spectra of the WCDs with excitation-dependent behavior are shown in Fig. 2(a) and (b). Compared with the polarity of AEAPMS, that of ethyl alcohol is relatively stronger. As mentioned above, the excitationdependent behavior is caused by the diverse surface states. It is worth mentioning that the two kinds of WCD in ethyl alcohol and AEAPMS solvents have different recombination processes, which are illustrated in Fig. 2(c).

As a result, ethyl alcohol appears to benefit the aggregation of the CDs, leading to the reduction of the PL QY from $57.1 \%$ to $50.8 \%$. This aggregation will increase energy losses and reduce the QY of the CDs. ${ }^{22}$ According to the above analysis, Fig. 2(c) illustrates the differences between the two kinds of WCD. For the pure RCDs, the particles could be retained among the solution. After ethyl alcohol is injected, the solution environment is changed due to the polarity. Similarly, the solution environment in AEAPMS is also altered by the protonation and deprotonation of the CDs. ${ }^{19}$ This process happens quickly, resulting in an increase of the viscosity of the mixed solutions. Specifically, amine-based and oxygen-based groups in the organosilane groups might be attracted to the surface states in the CDs. Therefore, excitation-dependent behavior of the WCDs was observed, which is due to the diverse surface groups. Fig. S6(a) and (b) $\dagger$ show the PL spectra $\left(\lambda_{\text {ex }}=400 \mathrm{~nm}\right)$ of the WCDs dispersed in AEAPMS (solid state) for a variety of storage times and a graph of the fluorescence intensity for a variety of storage times $\left(\lambda_{\mathrm{ex}}=400 \mathrm{~nm}\right)$. On increasing the storage time, there is nearly no decrease of the fluorescence intensity or shifting of the PL peaks. Therefore, we can conclude that the obtained RCDs dispersed in AEAPMS have a favorable photostability.

Although the fluorescence mechanism is unclear at present, it continues to motivate researchers to conduct deeper investigation. This may be attributed to quantum size effects and the surface defects on the CDs according to previous reports. ${ }^{2,3,23}$ In our case, we believe the surface states to be the primary factor considering the spectroscopy results along with the TEM images (Fig. 1(a)). Solvent-dependent photoluminescence is considered as a dipole induced surface electronic state change. The excited states on the CD surface are strongly influenced by the dipoles of the solvents. ${ }^{24,25}$ As shown in Fig. S7, $\dagger$ for the CDs with various surface states, except for the HOMO-2 $(\pi)$ energy level, two new HOMO-1 and HOMO energy levels are introduced by the abundant surface functional groups, such as $\mathrm{C}=\mathrm{O}$ and $\mathrm{C}=\mathrm{N}$. Then, efficient electron transitions can occur in the two new HOMO-1 and HOMO energy levels to the LUMO $\left(\pi^{*}\right)$ energy level. Shifting of the PL band to the long wavelength region will occur while the electrons in the N-related defect states return to the HOMO. ${ }^{4}$ The WCDs were produced due to the surface groups. More importantly, the multiple excitation centres on every nanoparticle and surface energy trap are created by the surface groups. ${ }^{17}$ It is believed that the surface 

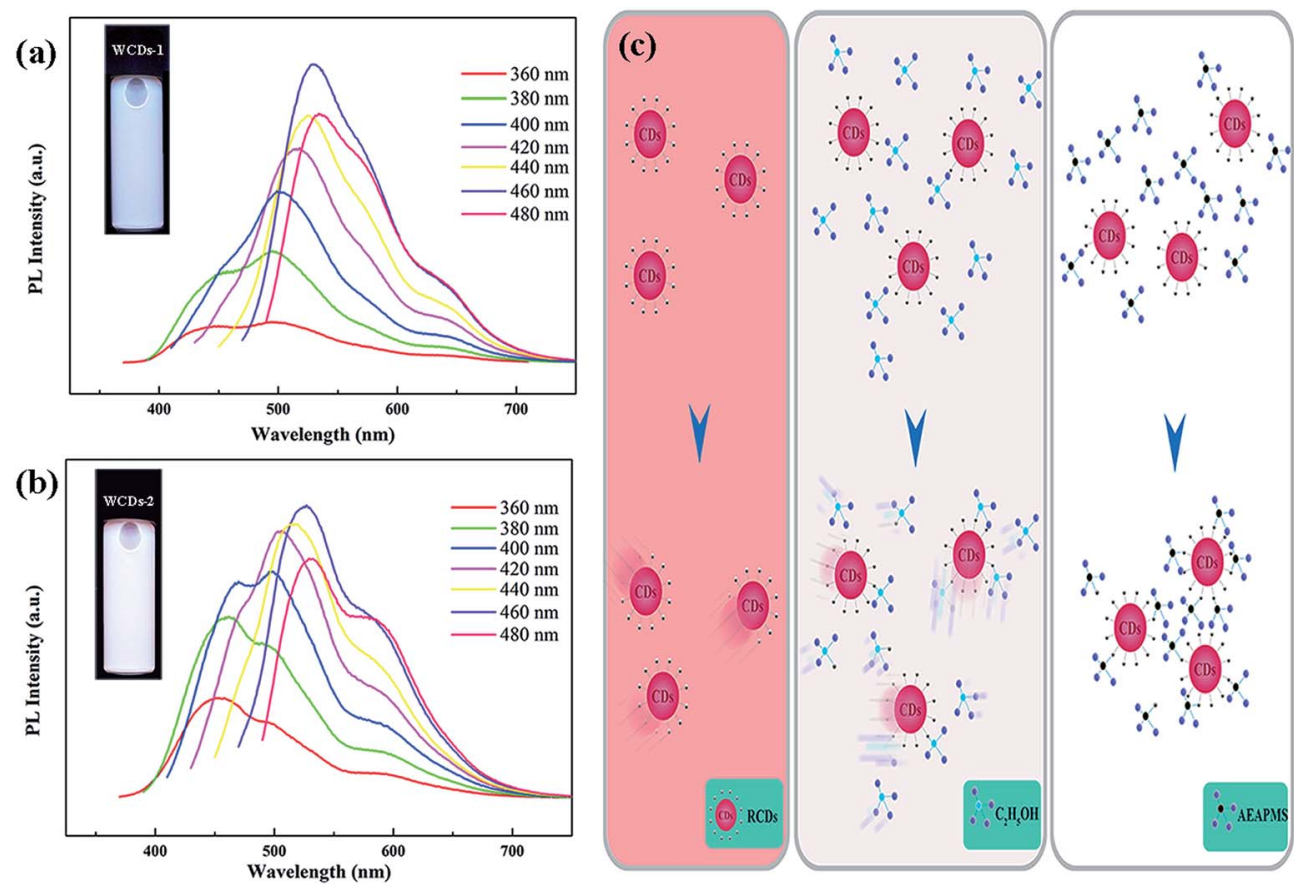

Fig. 2 PL emission spectra of the WCDs-1 (a) and WCDs-2 (b). (c) Schematics of the formation of the RCDs dispersed in ethyl alcohol and AEAPMS.

states of the CDs are similar to the molecular states, wherein various surface states correspond to a relatively wide distribution of different energy levels. The emission band shape of the WCDs is asymmetric with small shoulder peaks in the visible region. These phenomena suggest that the PL of the WCDs is related to the diverse surface states. Similarly, the absorption spectra of the WCDs (Fig. S8 $\dagger$ ) exhibit a broad band in the region of 250-500 nm, implying that multiple energy levels exist in the CDs. The white light may be attributed to the overlap of diverse light emissions induced by the electron transitions in the multiple levels. It is believed that the surface states of CDs doped in ethanol and AEAPMS are analogous to the molecular states. The various surface states correspond to a relatively wide distribution of different energy levels, which extends the excitation wavelength range and causes the excitation-dependent behavior. These oxygen and nitrogen structures increase the charge carrier density and induce the charge transfer of electrons towards the different energy levels. The white fluorescence
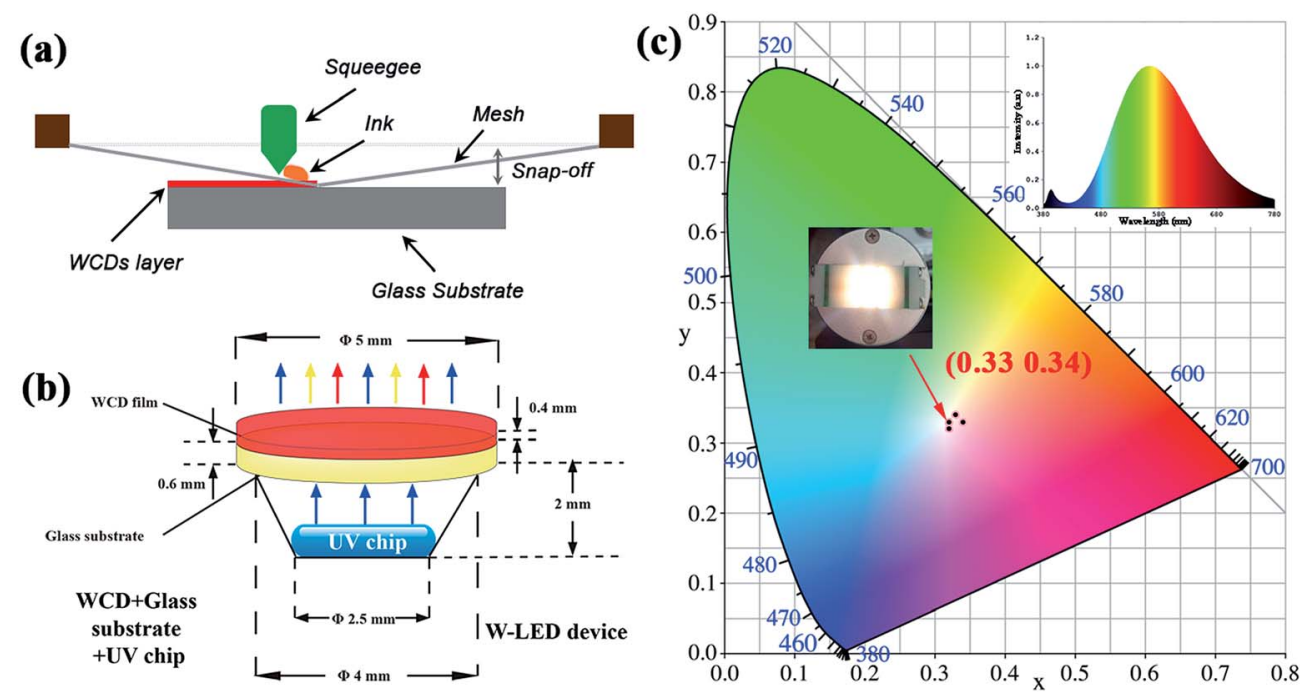

Fig. 3 (a) Schematic of the fabrication with screen-printing. (b) Schematic diagram of the W-LED from the combination of a UV LED chip with the superposed WCD layers. (c) The CIE coordinates of the WCD-based LED devices. Insets show the corresponding EL spectra and the luminescence photographs in operation. 
Table 1 Photoelectric parameters of the W-LEDs

\begin{tabular}{|c|c|c|c|c|c|c|}
\hline \multirow[b]{2}{*}{ Samples } & \multirow[b]{2}{*}{ Thickness } & \multicolumn{2}{|c|}{$\begin{array}{l}\text { CIE } \\
\text { coordinates }\end{array}$} & \multirow{2}{*}{$\begin{array}{l}\mathrm{LE}(\operatorname{lm} \\
\left.\mathrm{W}^{-1}\right)\end{array}$} & \multirow[b]{2}{*}{ CCT (K) } & \multirow[b]{2}{*}{ CRI } \\
\hline & & $X$ & $Y$ & & & \\
\hline WCDs-1 & $0.2 \mathrm{~mm}$ & 0.33 & 0.34 & 25.63 & 6735 & 51.0 \\
\hline WCDs-2 & & 0.32 & 0.32 & 25.37 & 6794 & 50.8 \\
\hline WCDs-3 & & 0.32 & 0.33 & 25.50 & 6780 & 50 \\
\hline WCDs-4 & & 0.34 & 0.33 & 25.58 & 6784 & 50 \\
\hline WCDs-5 & & 0.33 & 0.34 & 25.61 & 6737 & 50.8 \\
\hline
\end{tabular}

mechanism of the CDs revealed a multilevel emissive recombination channel, which might be from the distribution of diverse surface-state PL centers. In this case, the presence of alcohol and AEAPMS might further promote the increase of the QY of the WCDs, which is consistent with our test. Therefore, the overlapping of emissions by multiple levels is proposed to explain the white fluorescence.

Notably, the silane-amine based CDs have a great filmforming ability. ${ }^{6}$ Moreover, the organosilane could hold the WCDs to avoid the aggregation of the WCDs. The QY of the white CD-based glass thin-film was measured to be $56.3 \%$. Hence, the WCDs- 2 are a satisfactory candidate material. To investigate the potential application in W-LEDs, a low-cost screen-printing technique was used to form a homogeneous WCD layer on the glass plate, as displayed in Fig. 3(a). Meanwhile, the construction of a W-LED by combining the hybrid of the WCD film and glass matrix together with a commercially available UV LED (centered at $395 \mathrm{~nm}$ ) is illustrated in Fig. 3(b). The related electroluminescence (EL) spectrum of the constructed LED presents an emission band peaking at $395 \mathrm{~nm}$ from the UV chip and a broad white emission band from the WCD film, as shown in Fig. 3(c). To validate the reproducibility of the parameters of the obtained W-LED, we have tested these with extensive experiments. After multiple tests, the optimal photoelectric parameters are a CIE of $(0.33,0.34)$, a CCT of 6735 $\mathrm{K}$ and a CRI of 51 , as shown in Table 1 . These results demonstrate that the CDs provide a significant advancement in $\mathrm{W}$ LEDs.

\section{Conclusion}

In summary, excellent WCDs emitting bright white light with a QY of 57.1\% have been developed. A series of characterizations have been implemented to confirm the structure and properties of the CDs. To our knowledge, this is the first report on RCDs displaying white fluorescence in an alcohol and AEAPMS. The excitation-dependent behavior and solvatochromism phenomenon of the RCDs result from the diverse surface defect sites. Efficient and stable WCDs in AEAPMS were produced, which confirms that AEAPMS is a great passivator to avoid the fluorescence quenching of CDs. Furthermore, the excited states on the CD surface are strongly influenced by the dipoles of the solvents, which can promote the QY of the CDs. White fluorescence was achieved from the overlapping of emissions by multiple levels. Moreover, feasible white CD-based glass thin-films were constructed. The optimal W-LED exhibited a CIE of $(0.33,0.34)$, with a CRI of 51 at a CCT of $6735 \mathrm{~K}$. This demonstrates that the efficient and stable WCDs would be a promising candidate material for W-LEDs.

\section{Conflicts of interest}

There are no conflicts to declare.

\section{Acknowledgements}

The work was financially supported by the National Natural Science Foundation of China (No. 51472183 and 51672192) and the Zhejiang Province Natural Science Foundation (LQ14E020006).

\section{References}

1 S. N. Baker and G. A. Baker, Angew. Chem., Int. Ed., 2010, 49, 6726-6744.

2 S. Zhu, Y. Song, X. Zhao, J. Shao, J. Zhang and B. Yang, Nano Res., 2015, 8, 355-381.

3 Z. L. Wu, Z. X. Liu and Y. H. Yuan, J. Mater. Chem. B, 2017, 5, 3794-3809.

4 D. Chen, W. Wu, Y. Yuan, Y. Zhou, Z. Wan and P. Huang, J. Mater. Chem. C, 2016, 4, 9027-9035.

5 C. Sun, Y. Zhang, S. Kalytchuk, Y. Wang, X. Zhang, W. Gao, J. Zhao, K. Cepe, R. Zboril, W. Yu and A. Rogach, J. Mater. Chem. C, 2015, 3, 6613-6615.

6 Y. Zhang, J. Zhang, J. Zhang, S. Lin, Y. Y. Huang, R. R. Yuan, X. J. Liang and W. D. Xiang, Dyes Pigm., 2017, 140, 122-130.

7 F. Wang, M. Kreiter, B. He, S. Pang and C. Liu, Chem. Commun., 2010, 46, 3309-3311.

8 X. Guo, C. F. Wang, Z. Y. Yu, L. Chen and S. Chen, Chem. Commun., 2012, 48, 2692-2694.

9 H. Dang, L. K. Huang, Y. Zhang, C. F. Wang and S. Chen, Ind. Eng. Chem. Res., 2016, 55, 5335-5341.

10 K. Jiang, L. Zhang, J. Lu, C. Xu, C. Cai and H. Lin, Angew. Chem., Int. Ed., 2016, 55, 7231-7235.

11 H. Wang, C. Sun, X. Chen, Y. Zhang, V. Colvin, Q. Rice, J. Seo, S. Feng, S. Wang and W. Yu, Nanoscale, 2017, 9, 1909-1915.

12 S. Zhu, J. Zhang, C. Qiao, S. Tang, Y. Li, W. Yuan, B. Li, L. Tian, F. Liu, R. Hu, H. Gao, H. Wei, H. Zhang, H. Sun and B. Yang, Chem. Commun., 2011, 47, 6858-6860.

13 Y. Chen, M. Zheng, Y. Xiao, H. Dong, H. Zhang, J. Zhuang and Y. Liu, Adv. Mater., 2016, 28, 312-318.

14 J. He, Y. He, Y. Chen, B. Lei, J. Zhuang, Y. Xiao and Y. Liu, Small, 2017, 13, 170075.

15 Y. He, J. He, L. Wang, Z. Yu, H. Zhang, Y. Liu and B. Lei, Sens. Actuators, B, 2017, 251, 918-926.

16 T. T. Meiling, P. J. Cywiński and I. Bald, Sci. Rep., 2016, 6, 28557-28566.

17 L. H. Mao, W. Q. Tang, Z. Y. Deng, S. S. Liu, C. F. Wang and S. Chen, Ind. Eng. Chem. Res., 2014, 53, 6417-6425.

18 F. Wang, Z. Xie, H. Zhang, C. Y. Liu and Y. G. Zhang, Adv. Funct. Mater., 2011, 21, 1027-1031. 
19 Y. Dong, H. Pang, H. Yang, C. Guo, J. Shao, Y. Chi, C. Li and T. Yu, Angew. Chem., Int. Ed., 2013, 52, 7800-7804.

20 P. Yang, J. Zhao, L. Zhang, L. Li and Z. Zhu, Chem.-Eur. J., 2015, 21, 8561-8568.

21 H. Ding, S. Yu, J. Wei and H. Xiong, ACS Nano, 2015, 10, 484491.

22 D. Zhou, D. Li, P. Jing, Y. Zhai, D. Shen, S. Qu and A. Rogach, Chem. Mater., 2017, 29, 1779-1787.
23 S. Hu, Chem. Rec., 2016, 16, 219-230.

24 F. Terenziani, A. Painelli, C. Katan, M. Charlot and M. Blanchard-Desce, J. Am. Chem. Soc., 2006, 128, 1574215755.

25 P. K. Lekha and E. Prasad, Chem.-Eur. J., 2011, 17, 86098617. 